Research Article

\title{
The Efficacy of Concentrated Growth Factor in the Healing of Alveolar Osteitis: A Clinical Study
}

\author{
Aqsa Kamal, ${ }^{1,2}$ Basheer Salman, ${ }^{1}$ Noor Hayati Abdul Razak, ${ }^{2}$ Ali Al Qabbani, ${ }^{1}$ \\ and A. R. Samsudin $\mathbb{( D D}^{1}$ \\ ${ }^{1}$ College of Dental Medicine, University of Sharjah, Sharjah, UAE \\ ${ }^{2}$ School of Dental Sciences, Universiti Sains Malaysia, Penang, Malaysia \\ Correspondence should be addressed to A. R. Samsudin; drabrani@sharjah.ac.ae
}

Received 12 December 2019; Accepted 10 March 2020; Published 12 May 2020

Academic Editor: Leonzio Fortunato

Copyright (C) 2020 Aqsa Kamal et al. This is an open access article distributed under the Creative Commons Attribution License, which permits unrestricted use, distribution, and reproduction in any medium, provided the original work is properly cited.

Background. A dry socket also referred to as alveolar osteitis (AO) is a common postoperative complication following tooth extraction, due to the disruption of the clot within the wound. This study aimed to evaluate the efficacy of concentrated growth factor (CGF) in the healing of alveolar osteitis following tooth extraction. Methods. The study was conducted at University Dental Hospital Sharjah, UAE. Patients undergoing tooth extraction at the oral surgery clinic were advised to return immediately if they suffer from pain. Over the following first week after tooth extraction, patients who reported pain symptoms were recalled and all dry sockets were identified. The patients were divided into two groups. Group I patients received conventional treatment with socket curettage and saline irrigation only, while in group II CGF was inserted into the socket. Both groups were observed for pain score and quantification of granulation tissue formation. Results. A total of 40 dry socket patients, aged between 18 and 60 years, from a total of 1,250 patients, were included in the study. 30 patients were given conventional treatment while another 10 patients were given CGF. Patients who received CGF had a pain score of 7-10 at presentation, and the pain score dropped to 0-3 on day 4 and further improved to $0-1$ on day $7(p=0.001)$. Granulation tissue formation appeared in the conventional group I on day 7 while the CGF group II showed earlier granulation tissue formation by day 4 ( $p=0.001$ ). The posttreatment pain score is inversely proportional to the amount and rate of granulation tissue formation in the socket. Conclusion. The study suggests that delivery of CGF into a dry socket helps relieve pain and expedite the wound healing process as shown by a statistically much lower pain score and earlier and more rapid formation of granulation tissue when compared to the conventional alveolar osteitis therapy.

\section{Introduction}

A dry socket also referred to as alveolar osteitis is a postoperative complication following tooth extraction. Both terms "dry socket" and "alveolar osteitis" have been used interchangeably in the dental literature. It can be defined as "postoperative pain within and around the extraction site, which rises in severity at any time between the first and fifth days after the tooth extraction, accompanied by a partial or complete disintegration blood clot within the alveolar socket and with or without halitosis" [1]. It occurs in $0.5-5 \%$ of routine dental extractions and $25-30 \%$ following the extraction of impacted mandibular wisdom tooth $[2,3]$. Females are more frequently affected than males, but this appears to be related to oral contraceptive use rather than any underlying gender predilection [4]. Unlike other forms of wound infection, alveolar osteitis occurs frequently in the young age group, although wound infection, in general, is more likely to occur with increasing age.

Even, in this era of cell and molecular biology, the specific etiology of the dry socket has not yet been defined. However, numerous local and systemic elements make contributions towards it $[1,5,6]$. Some of the risk factors are difficult surgical extraction, trauma, microbiological origin, smoking, age, and contraceptive pill use [7, 8]. Presence of periodontal disease, acute necrotizing ulcerative gingivitis, local bone disease, or previous history of developing a dry socket has also been implicated $[3,9,10]$. 
Clinical and experimental research studies have described an elevated fibrinolytic activity as a major factor for the etiology of the dry socket $[6,11-14]$. Human plasminogen is a single-chain glycoprotein containing 791 amino acid residues and $2 \%$ carbohydrate. In these studies, plasminogen type-1, released following a routine tooth extraction, is the precursor of plasmin, a potent serine protease involved in the dissolution of fibrin clots (Figure 1). Type-1 plasminogen appears more readily recruited to blood clots $[6,16]$. Tissue-type plasminogen activator ( $\mathrm{t}-\mathrm{PA}$ ) is the principal endogenous activator of plasminogen in blood. It is produced as a singlechain molecule by the vascular endothelial cells and is secreted into the plasma by an acute release following stimulation of certain endothelial cell receptors such as in tooth extraction injury. It is also known that plasmin deficiency may lead to thrombosis, as clots are not adequately degraded. Plasmin activity is inhibited mainly by binding to the plasmin inhibitor, which forms a stable complex with plasmin devoid of proteolytic activity $[6,16]$.

Being an old surgical puzzle, many different methods have been advocated to treat alveolar osteitis, such as the application of local turmeric [17], zinc oxide eugenol, alvogyl, honey or vitamin C [18], and socket irrigation with hydrogen peroxide [19]. However, these conventional treatment approaches merely solve the symptoms but do not target the key etiology. Furthermore, most of these approaches do not completely alleviate the pain and other symptoms. More recent approaches come into play based on experience of wound healing management in other surgical specialty areas, and these include the use of low intensity pulsed ultrasound therapy (LIPUS) [20], low level laser therapy (LLLT) [21, 22], ozone therapy $[23,24]$, and the use of platelet rich plasma (PRP) in general and oral wound healing.

As the knowledge on the biology of wound healing advances, the role of cytokines and growth factors in the healing of alveolar osteitis becomes more significant [25]. Understanding the molecular aspects of wound healing plays an important role in dry socket healing. Over the past two decades, platelet rich plasma (PRP) has been used in many surgical fields as an additional remedy for supporting wound healing. Platelet concentrates are attractive autologous scaffolds suitable for regenerative medicine with its fiber architecture and rich growth factors [26]. Concentrated growth factor (CGF) is the third generation of autologous plasma extract prepared by a special centrifugal program [27]. Its application in the oral region is still controversial particularly when oral wound healing shows different immunologic responses and regenerative capabilities compared to general surgical wounds $[28,29]$. Therefore, the aim of this study is to investigate the efficacy of CGF as a local treatment option for alveolar osteitis (AO) compared with conventional therapy.

\section{Materials and Methods}

This study was conducted from August till October 2019 at University Dental Hospital Sharjah (UDHS), United Arab Emirates. Human ethics approval was obtained from the Research Ethics Committee University of Sharjah REC-17-
02-14-01-S dated $24^{\text {th }}$ October, 2017. The study population was patient treatment at the oral surgery clinic of University Dental Hospital Sharjah. The subjects included were patients who underwent routine tooth extractions performed under local anesthesia following the diagnosis of irreversible pulpitis, chronic and acute apical pathology, periapical abscesses, and advance periodontitis. Other reasons for tooth extractions and the presence of local odontogenic tumors or malignancies were excluded. Patients with wellcontrolled systemic medical conditions such as diabetes and hypertension were included in the study. Following tooth extraction, the socket was curetted in cases of periapical pathology and patients were asked to bite on a piece of sterile gauze for 30 minutes to achieve hemostasis. There were no additional dressings or medication inserted in the socket. The presence of a nice healthy clot as observed by the attending dentist was confirmed before the patients were discharged from the clinic. They were advised to return immediately to the University Dental Hospital if they suffer from postoperative pain. Complicated tooth extractions under local anesthesia that lead to minor oral surgical procedures were excluded. All patients included in the study were not prescribed antibiotics. Over the following five days following the tooth extraction, the patients who reported suffering from postoperative pain symptoms were reexamined in the clinic.

Patients who were diagnosed with alveolar osteitis were referred to the oral surgery department. The selection of the subjects was done according to the previous inclusion and exclusion criteria. All patients aged between 18 and 60 years who were diagnosed with alveolar osteitis were included in the study. Those with good oral hygiene who had undergone nonsurgical tooth extraction under local anesthesia and who suffered postoperative pain between 1 and 5 postextraction days presenting with empty dry socket were selected. Patients aged below 18 and above 60 years and those who had tooth extraction performed outside UDHS or patients who underwent surgical extraction were excluded. The patient's pain level was recorded on a visual analogue scale of 1 to 10 $[30,31]$ (Figure 2), and their body temperature was also recorded.

The selected patients were divided into two groups based on their choice to be treated with either conventional treatment (group I) or CGF treatment (group II).

In group I the patients were treated with conventional treatment whereby the dry socket was curetted and irrigated with saline under local anesthesia. A new bleeding socket was created following curettage and gentle saline irrigations help debride the necrotic debris. The patient was instructed to bite on a piece of sterile gauge to achieve hemostasis.

In group II, the socket was similarly curetted and irrigated under local anesthesia and CGF in gel form was inserted into it. Preparation of CGF was performed by obtaining about $9 \mathrm{ml}$ of the patient's blood into a vacuum test tube. CGF was prepared using Medifuge centrifuge machine, Silfradent, Italy, following a cycle duration of 5 minutes at 1,000 revolutions. The processing time is about 12 minutes and finally a thick yellowish color gel layer was 


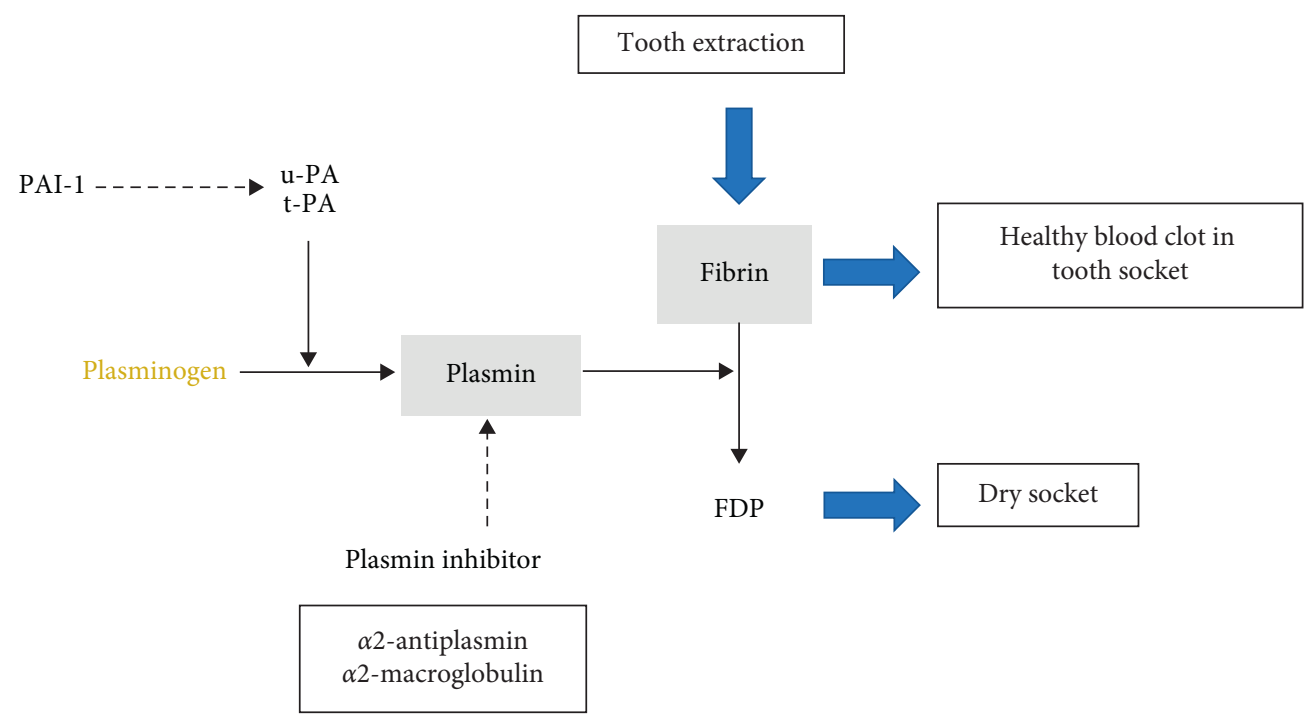

Figure 1: Molecular mechanism of alveolar osteitis. Plasminogen type-1 is the precursor of plasmin which acts in fibrin degradation. Plasmin inhibitor sterically shields the active site of plasmin, thus substantially decreasing plasmin's access to protein substrates [15]. $t-P A$ : tissue-type plasminogen activator; FDP: fibrin degradation products.

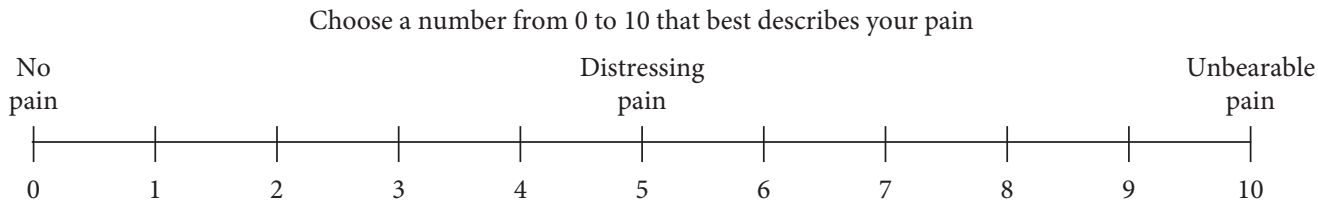

Figure 2: Visual analogue pain scale (VAS) for recording the pain level of patients for dry socket [30, 31].

produced known as CGF. This CGF gel was directly delivered into the socket using a surgical tweezer [32-34].

A periapical radiograph of the socket was done for both groups before initiating treatment to exclude the presence of retained apices, bony fragments, and fracture of the alveolus. No dressing such as alvogyl or topical antibiotic was placed in both groups and no systemic antibiotic was prescribed.

The day of the patient's return to the clinic with a dry socket presentation was recorded as "Day 0." Both groups were observed for pain score using a visual analogue pain scale from 1 to 10 (Figure 2) and quantification of clinically evident granulation tissue formation within the socket was recorded on day 0 , day 4 , and day 7 .

Continued follow-up of patients in both groups was done through phone calls on a weekly interval for up to 4 weeks after dry socket treatment.

2.1. Statistical Analysis. All the data was entered into SPSS software. We used Kruskal-Wallis test to determine the mean and standard deviation for age and scoring of pain and granulation tissue formation.

A $p$ value of less than 0.05 is considered significant.

\section{Results}

Over a three-month period, a total of 40 dry socket patients, 25 males and 15 females, were included in the study (see Table 1) from a total of 1,250 patients who attended for
TABle 1: Patient demography.

\begin{tabular}{lcc}
\hline & $\begin{array}{c}\text { Mean (standard } \\
\text { deviation, SD) }\end{array}$ & $\begin{array}{c}\text { Frequency } \\
\text { (percentage \%) }\end{array}$ \\
\hline Age & $38.03(8.96)$ & \\
Gender & & \\
Male & & $25(62.5)$ \\
Female & & $15(37.5)$ \\
\hline
\end{tabular}

routine tooth extraction under local anesthesia at oral surgery clinic, University Dental Hospital Sharjah. Among the dry socket cases, five patients were noted to be diabetic, four patients were hypertensive, three patients were both diabetic and hypertensive, and one patient had other medical conditions. Only three male patients were smokers and their ages were between 35 and 49 years. This study showed that more patients accepted the conventional treatment compared to the new CGF treatment. In group I, 30 patients aged between 18 and 60 were given conventional treatment while another 10 patients in group II aged between 18 and 60 were given CGF.

In the conventional group I, the pain score was 7-10 on the day of presentation (day 0 ) and the pain score dropped to 4-6 on day 4 and further decreased to 2-4 on day 7 (see Table 2) following treatment. Granulation tissue (GT) formation within the healing socket in group I appeared clearly only on day 7 . In group II patients who received CGF, a similar pain score of 7-10 was recorded on the day 
TABLe 2: Pain and granulation tissue (GT) score of subjects in group I and group II at days 0 , 4, and 7.

\begin{tabular}{|c|c|c|c|c|c|c|c|c|c|}
\hline \multirow{2}{*}{ Patient no. } & \multirow{2}{*}{ Age } & \multirow{2}{*}{ Gender } & \multirow{2}{*}{ Treatment option } & \multicolumn{2}{|l|}{ Day 0} & \multicolumn{2}{|c|}{ Day 4} & \multicolumn{2}{|c|}{ Day 7} \\
\hline & & & & Pain before treatment & GT & Pain & GT & Pain & GT \\
\hline 1 & 53 & Female & CGF & 7 & Nil & 0 & + & 0 & ++ \\
\hline 2 & 39 & Female & CGF & 8 & Nil & 0 & ++ & 0 & +++ \\
\hline 3 & 28 & Male & Control & 10 & Nil & 6 & Nil & 3 & + \\
\hline 4 & 35 & Male & Control & 9 & Nil & 5 & + & 5 & + \\
\hline 5 & 38 & Male & Control & 8 & Nil & 6 & Nil & 3 & + \\
\hline 6 & 33 & Male & CGF & 9 & Nil & 1 & ++ & 0 & +++ \\
\hline 7 & 38 & Male & CGF & 8 & Nil & 0 & ++ & 0 & ++ \\
\hline 8 & 29 & Male & CGF & 9 & Nil & 0 & + & 0 & +++ \\
\hline 9 & 40 & Female & Control & 10 & Nil & 5 & Nil & 3 & + \\
\hline 10 & 49 & Female & Control & 8 & Nil & 4 & Nil & 3 & + \\
\hline 11 & 42 & Male & Control & 9 & Nil & 5 & Nil & 0 & + \\
\hline 12 & 40 & Female & CGF & 9 & Nil & 1 & ++ & 0 & +++ \\
\hline 13 & 38 & Female & CGF & 7 & Nil & 0 & + & 0 & +++ \\
\hline 14 & 40 & Male & CGF & 7 & Nil & 0 & ++ & 0 & ++ \\
\hline 15 & 35 & Male & Control & 8 & Nil & 4 & Nil & 0 & + \\
\hline 16 & 32 & Female & Control & 8 & Nil & 5 & Nil & 4 & + \\
\hline 17 & 49 & Male & CGF & 8 & Nil & 1 & ++ & 0 & ++ \\
\hline 18 & 31 & Male & CGF & 8 & Nil & 0 & ++ & 0 & +++ \\
\hline 19 & 42 & Female & Control & 7 & Nil & 3 & Nil & 5 & + \\
\hline 20 & 36 & Male & Control & 8 & Nil & 5 & Nil & 3 & + \\
\hline 21 & 27 & Male & Control & 10 & Nil & 6 & Nil & 4 & + \\
\hline 22 & 42 & Female & Control & 8 & Nil & 5 & + & 3 & + \\
\hline 23 & 36 & Male & Control & 9 & Nil & 6 & Nil & 4 & + \\
\hline 24 & 51 & Male & Control & 8 & Nil & 4 & Nil & 3 & + \\
\hline 25 & 35 & Female & Control & 10 & Nil & 6 & + & 3 & + \\
\hline 26 & 49 & Male & Control & 9 & Nil & 7 & Nil & 2 & + \\
\hline 27 & 60 & Male & Control & 9 & Nil & 6 & Nil & 3 & + \\
\hline 28 & 40 & Female & Control & 8 & Nil & 5 & Nil & 3 & + \\
\hline 29 & 47 & Male & Control & 8 & Nil & 7 & Nil & 2 & + \\
\hline 30 & 40 & Female & Control & 7 & Nil & 7 & Nil & 4 & + \\
\hline 31 & 57 & Male & Control & 10 & Nil & 6 & + & 4 & ++ \\
\hline 32 & 36 & Female & Control & 8 & Nil & 6 & Nil & 4 & + \\
\hline 33 & 23 & Male & Control & 9 & Nil & 6 & + & 4 & ++ \\
\hline 34 & 23 & Male & Control & 8 & Nil & 5 & Nil & 4 & + \\
\hline 35 & 27 & Male & Control & 8 & Nil & 4 & Nil & 3 & ++ \\
\hline 36 & 23 & Male & Control & 8 & Nil & 4 & Nil & 2 & + \\
\hline 37 & 40 & Female & Control & 10 & Nil & 4 & + & 2 & ++ \\
\hline 38 & 38 & Male & Control & 10 & Nil & 5 & + & 3 & ++ \\
\hline 39 & 30 & Male & Control & 9 & Nil & 4 & Nil & 3 & + \\
\hline 40 & 30 & Female & Control & 9 & Nil & 4 & Nil & 2 & + \\
\hline
\end{tabular}

of presentation (day 0), and the pain score dropped to 0-3 on day 4 and further improved to $0-1$ on day 7 (see Table 2).

Quantification of clinically evident granulation tissue formation within the socket was recorded (see Table 3), from day 0 , day 4, and day 7 after dry socket treatment. A completely barren dry socket without granulation tissue GT was recorded as (nil). The formation of GT in one quarter or less of the socket was recorded as $(+)$, while formation of granulation tissue in half of the socket was recorded as $(++)$, formation of granulation tissue in three quarters was recorded as $(+++)$, and complete laying down of granulation tissue within the whole socket was recorded as $(++++)$.

The CGF applied sockets among group II patients also showed much earlier granulation tissue GT by day 4 . By the second week postoperatively, both groups showed similar pain score and GT formation and showed comparable wound healing stage after 14 days of post dry socket treatment. The results were tested statistically (see Tables 4 and 5) to see the significance.

\section{Discussion}

In this study, the incidence of the dry socket in University Dental Hospital Sharjah is around 3\%. The incidence of dry socket has been reported as 1-4\% world wide following routine dental extractions, with the incidence increasing to 10 times greater for lower as compared to upper teeth, and reaching $45 \%$ for mandibular third molar removal [1, 35]. Our data is quite consistent with many other studies and it is interesting to note that dry socket incidence does not seem to run parallel with clinical standards of infection control since our infection control protocol is much higher than many other centers that report the same incidence. This fact may 
TABLE 3: Scoring system employed for granulation tissue (GT) formation in the dry socket.

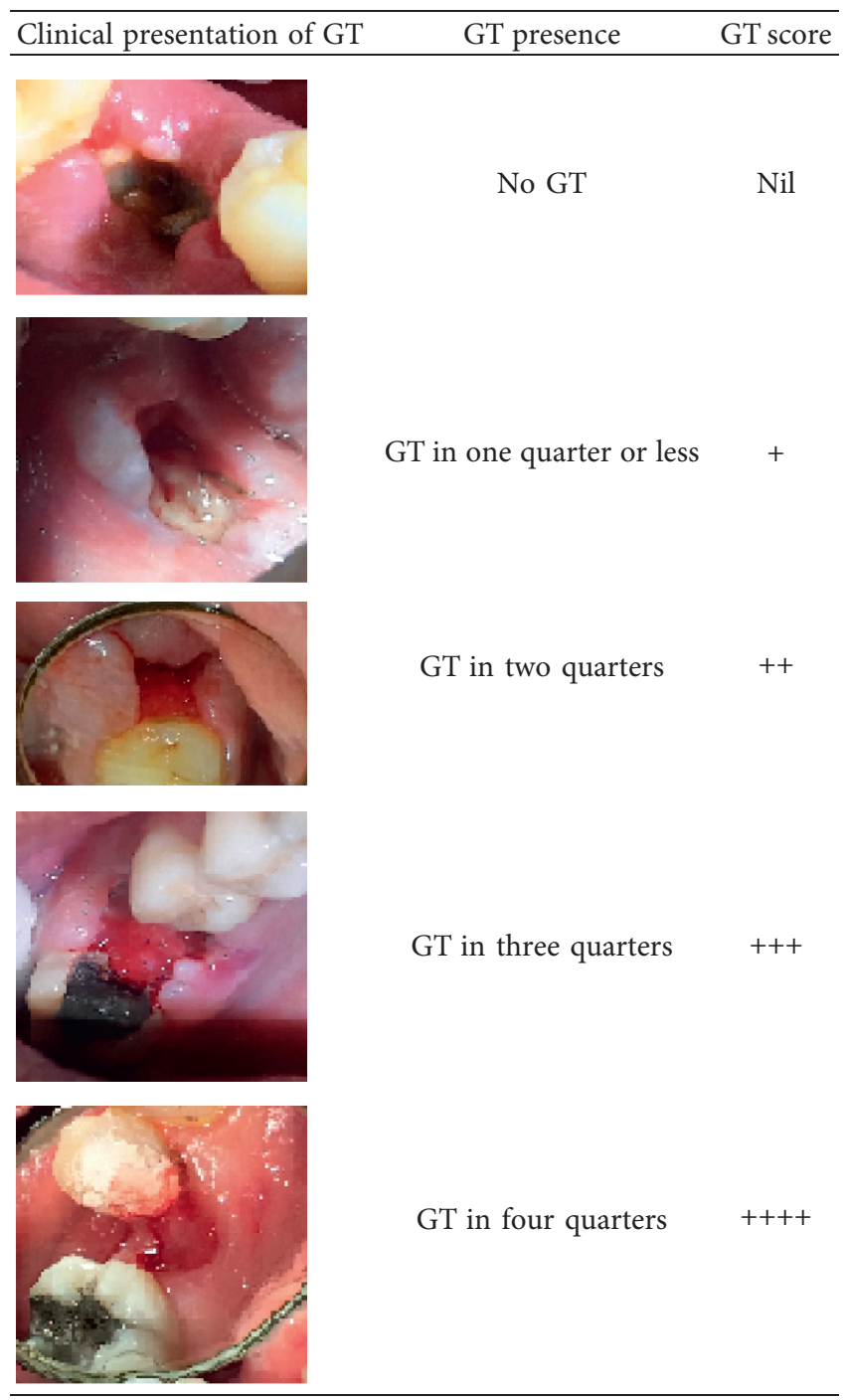

TABLe 4: Pain score among groups I and II.

\begin{tabular}{llcc}
\hline Day & Group & Mean rank & $p$ value \\
\hline \multirow{2}{*}{ Day 0 } & Group I & 22.37 & 0.065 \\
& Group II & 14.90 & 0.001 \\
\multirow{2}{*}{ Day 4 } & Group I & 25.50 & \multirow{2}{*}{0.50} \\
\hline \multirow{2}{*}{ Day 7 } & Group II & 25.17 & \multirow{2}{*}{6.001} \\
\hline
\end{tabular}

suggest a controversy against the microbial etiology for dry sockets.

There are more dry socket subjects within the middle age group among the 40 patients seen at University Dental Hospital Sharjah. The mean age of dry socket subjects was 38.03 years. More male patients were seen with dry sockets although the University Dental Hospital records show almost an equal number of both male and female patients undergoing tooth extraction on a daily basis. Interestingly, male was predominantly ranging from 30 to 49 years and less females were seen, mostly in the age range from 30 to 49
TABLE 5: Granulation tissue score in groups I and II.

\begin{tabular}{llcc}
\hline Day & Group & Mean rank & $p$ value \\
\hline \multirow{2}{*}{ Day 4 } & Group I & 16.08 & 0.001 \\
& Group II & 33.75 & \\
\hline \multirow{2}{*}{ Day 7 } & Group I & 15.83 & 0.001 \\
& Group II & 34.50 & \\
\hline
\end{tabular}

years. The lesser number of females may be due to lower contraceptive drug consumption in the United Arab Emirates [36], since more than $50 \%$ of women in childbearing age do not favour contraception, and this factor may contribute to a lower number of alveolar osteitis among women [36]. Use of oral contraceptives has been shown to significantly increase the risk of dry socket since estrogens and other risk factors such as surgical trauma are thought to cause dry socket by stimulating fibrinolysis $[37,38]$.

Incidence of alveolar osteitis commonly occurs among patients aged 18 years or older as compared to younger individuals $[4,6]$. Dry socket rarely occurs in children and some other etiology must be suspected if it happens in the pediatric age group [39]. Some studies suggest that alveolar osteitis rarely occurs during childhood, because this is a period in which micro-organisms such as Treponema denticola are usually not detected in the oral environment, and such bacteria have not colonized in the pediatric oral cavity [8]. Young adults and middle-aged individuals are more prone to dry sockets due to a much higher immunological response to injury compared to the older age group. Some studies suggest that age has an inverse relation with the formation of alveolar osteitis [35] which is in contrast to our findings since there were very few patients who were in 18-29 years of the age range that suffer from alveolar osteitis.

We noted that all patients suffer severe pain with a VAS pain score of 7-10 on the day of presentation. The lysis of the blood clot in the socket leads to fibrin degradation products attributed to the formation of kinins in the compact socket. Plasmin is involved in the conversion of kallikrein into kinins and together with other inflammatory mediators causes intense pain. Following conventional treatment under local anesthesia, the pain score dropped to VAS 4-6 on day 4 and clinical findings showed some laying down of GT formation within the socket. In the sockets treated with CGF, a much more drastic improvement in pain symptom was shown with a VAS of $0-3$ as early as day 4 , and clinical evaluation showed a much richer pool of granulation tissue within the healing socket. This observation demonstrated that the amount and rate of granulation tissue (GT) formation in the socket are inversely proportional to the pain score and there is a significant effectiveness of CGF on the healing of dry socket and improvement in symptoms $(p=0.001)$.

None of the patients in both groups showed a rise in body temperature. This shows that alveolar osteitis is a local condition without systemic upset. This study captured a few dry socket patients with controlled diabetes and hypertension. All patients included in this study were in good health. Most studies suggest that systemic conditions play a vital 
role in the etiology of alveolar osteitis [40]. However, many authors do agree that both healthy and medically compromised patients with systemic conditions such as being diabetic may succumb to dry socket following normal tooth extraction. The question of whether medically compromised patients are more prone to suffer from dry sockets is difficult to quantify. Furthermore, our University Dental Hospital is a referral center for medically compromised dental patients but there were very few medically compromised patients who underwent tooth extraction and succumbed to a dry socket complication. It must be emphasized that only wellcontrolled diabetics and hypertensive patients are permitted to undergo elective tooth extractions at the University Dental Hospital Sharjah. Therefore, this study suggests that patients with well-controlled systemic diseases are good candidates for tooth extraction and there are yet other unknown factors that compromise early healing of the socket resulting in alveolar osteitis.

Smoking is known to interfere with wound healing. Only three smokers suffered from dry sockets in this study. There are different studies which suggest that smoking is a strong predisposing factor for the formation of the dry socket [41], although most subjects in this study deny current smoking. We did not take the past history of cigarette smoking since it should be noted that cigarette smoking is an expensive habit in UAE. It is reported that self-reported tobacco use in UAE is $36 \%$ among men and 3\% in women [42]. Smoking as a strong etiology also makes the incidence of dry sockets be lower among children and females too.

In the conventional treatment approach, local anesthesia is given to patients followed by curettage of the bare necrotic bony socket to induce bleeding, and the socket was irrigated with normal saline. This is done to induce clot formation, by subjecting the wound again through all the phases of normal wound healing beginning from hemostasis, acute inflammation, proliferation, maturation, and remodeling. Platelets aggregates in severed vessels trigger the clotting cascade and release essential growth factors and cytokines that are important for the initiation and progression of wound healing. The fibrin matrix resulting from this stabilizes the wound and provides a provisional scaffold for the wound healing process. Curettage of the socket followed by irrigation with physiological saline allows debridement of necrotic soft tissue and bone debris supporting the development of a vital base for the invasion of new capillaries, laying down of fibroblast matrix, and granulation tissue formation.

Curettage of the dry socket was performed in both groups of patients. However, there are advocators who do not favour socket curettage for fear of inflicting further trauma [43]. Failure of adequate socket debridement will not favour the early invasion of fibrovascular tissue into the poorly vascularized wound and dependence on natural phagocytosis will take an extremely lengthy healing period.

Treatment with CGF showed a higher potency of the healing process. Concentrated growth factors are ideal for clotting as it contains essential growth factor: platelet-derived growth factor (PDGF), transforming growth factor (TGF), platelet factor interleukin (IL), vascular endothelial growth factor (VEGF), epidermal growth factor (EGF), insulin-like growth factor IGF, and fibronectin [34]. Together, this cocktail of growth factors speeds up the development of the delicate fibrovascular granulation tissue. Different studies have shown that the presence of growth factors can speed up the healing process [44, 45]. CGF promotes cell proliferation and migration and regulates the biological behavior of diverse cell types and supports angiogenesis which is a key element in any wound healing process [27].

In this study, patients were advised to come back immediately in case of any pain following the tooth extraction. Most patients reported having pain after 4-7 days. However, all patients started to have pain from the very next day after the tooth extraction but due to their job circumstances, no leave from work, family commitments, and other factors they tend to return to the clinic after few days. It was observed that acute pain develops almost immediately following lysis of the clot and exposure of the unprotected dry bony socket and nerve endings, with the production of kinins and inflammatory mediators. Almost all the patients had a pain score from 7 to 10 on the visual analogue pain scale associated with tenderness of the regional lymph nodes. The severe pain from the dry socket is often underestimated by the attending dentist.

An interesting observation in this study is the absence of pus discharge from the socket and afebrile status of a patient's body temperature. Many dental practitioners may misinterpret the yellowish necrotic bone slough as pus discharge from the socket. The absence of a rise in body temperature also signifies a dry socket to be a local wound dehiscence event without systemic physiological upset. The conventional treatment approach for the dry socket helps alleviate the pain through the formation of granulation tissue but recovery was slow. This phenomenon suggests that reformation of the natural blood clot (or some may call them secondary clot) in the treated dry socket is not as effective as the first natural clot formed immediately after tooth extraction. The addition of CGF will enhance the compromised weaker secondary clot. CGF gel is known to have closely interwoven fibers exhibiting a relatively stiffer texture than the traditional platelet rich fibrin (PRF) and able to promote cell adhesion and migration [46] during the proliferative healing phase.

Rutkowski et al., in 2010, used PRP to promote bone formation following extraction [33]. They observed the positive effects of PRP on bone formation and wound healing. Haraji et al., in 2012, also worked on dry socket treatment. They treated patients with PRP and showed that their beneficial properties are very effective in the treatment of dry sockets and in normal healthy extraction sockets including bone healing [32]. Chenchev et. al., in 2017, worked on alveolar osteitis using platelet rich fibrin (PRF). They too claimed that PRF can successfully treat dry socket by reducing pain symptoms and expedite wound closure and epithelialization [15, 45]. King et al. [47] worked on PRP as plasma rich in growth factors, to treat alveolar osteitis in 2018. They claimed that plasma rich in growth factors indeed has considerable advantages [47]. 
Granulation tissue is young connective tissue possessing microscopic blood vessels which is formed on the surface of a wound during the healing process, forming a protective layer, and resistant to microbial invasion. It is friable and may be easily damaged, but CGF seems to promote the formation and protection of granulation tissue in a compromised wound. This could be the main reason for the dry socket healing expedition and reduction of pain.

Our study showed that conventional dry socket treatment does provide palliation but the addition of CGF expedites the healing process and alleviates the symptoms much earlier [34]. It further demonstrates that conventionally treated sockets take more than 7 days to match the effective healing of a CGF treated socket. CGF works at the molecular level, targeting some of the key physiological deficiencies of the dry socket [32-34]. The use of autologous platelet concentrates may be helpful because of their local immunomodulatory properties and possible promotion of angiogenesis and tissue healing by platelet factors [48]. CGF, like other second-generation autologous platelet concentrates, is easy to prepare, is of low cost, and has minimal risk for the patient [49].

\section{Conclusion}

This study suggests that the delivery of CGF into a dry socket helps to relieve pain and expedite the wound healing process as shown by a much lower pain score and earlier and more rapid formation of granulation tissue when compared to conventional therapy. Chairside CGF techniques in the dental office are simple, feasible, and economical with predictable results.

\subsection{Observed Learning Points}

(i) Pain from the dry socket is excruciating and often underestimated by the attending dentist

(ii) Dry socket is a localized failure of tooth extraction wound healing

(iii) It causes neither a systemic upset nor a rise in body temperature

(iv) Low contraceptive consumption in the community tends to produce a lower prevalence of dry sockets among women in childbearing age

(v) Dry socket does not occur in children

(vi) Concentrated growth factor supports dry socket healing by expediting granulation tissue formation and alleviating pain

\section{Consent}

In this case report, the patient is sufficiently anonymized according to ICMJE guidelines.

\section{Conflicts of Interest}

The authors declare that there are no conflicts of interest regarding the publication of this article.

\section{References}

[1] I. R. Blum, "Contemporary views on dry socket (alveolar osteitis): a clinical appraisal of standardization, aetiopathogenesis and management: a critical review," International Journal of Oral and Maxillofacial Surgery, vol. 31, no. 3, pp. 309-317, 2002.

[2] D. C. Bowe, S. Rogers, and L. F. Stassen, "The management of dry socket/alveolar osteitis," Journal of the Irish Dental Association, vol. 57, no. 6, pp. 305-310, 2011.

[3] S. Preetha, "An overview of dry socket and its management," IOSR Journal of Dental and Medical Sciences, vol. 13, no. 5, p. 2, 2014.

[4] M. Chiapasco, M. Crescentini, and G. Romanoni, "The extraction of the lower third molars: germectomy or late avulsion?" Minerva Stomatologica, vol. 43, no. 5, pp. 191-198, 1994.

[5] P. J. Vezeau, "Dental extraction wound management: medicating postextraction sockets," Journal of Oral and Maxillofacial Surgery, vol. 58, no. 5, pp. 531-537, 2000.

[6] C. L. Cardoso, M. T. V. Rodrigues, O. F. Júnior, G. P. Garlet, and P. S. P. De Carvalho, "Clinical concepts of dry socket," Journal of Oral and Maxillofacial Surgery, vol. 68, no. 8, pp. 1922-1932, 2010.

[7] V. Rakhshan, "Common risk factors of dry socket (alveolitis osteitis) following dental extraction: a brief narrative review," Journal of Stomatology, Oral and Maxillofacial Surgery, vol. 119, no. 5, pp. 407-411, 2018.

[8] D. W. Nitzan, "On the genesis of "dry socket"” Journal of Oral and Maxillofacial Surgery, vol. 41, no. 11, pp. 706-710, 1983.

[9] E. W. Odell, Clinical Problem Solving In Dentistry 3/e, Elsevier, Amsterdam, Netherlands, 2010.

[10] B. Daly, M. O. Sharif, T. Newton, K. Jones, and $\mathrm{H}$. V. Worthington, "Local interventions for the management of alveolar osteitis (dry socket)," The Cochrane Database of Systematic Reviews, vol. 12, Article ID CD006968, 2012.

[11] H. Birn, "Bacteria and fibrinolytic activity in "dry socket"” Acta Odontologica Scandinavica, vol. 28, no. 6, pp. 773-783, 1970.

[12] H. Birn and O. Myhre-Jensen, "Cellular fibrinolytic activity of human alveolar bone," International Journal of Oral Surgery, vol. 1, no. 3, pp. 121-125, 1972.

[13] H. Birn, "Fibrinolytic activity of alveolar bone in "dry socke"” Acta Odontologica Scandinavica, vol. 30, no. 1, pp. 23-32, 1972.

[14] H. Birn, "Etiology and pathogenesis of fibrinolytic alveolitis (“dry socket")," International Journal of Oral Surgery, vol. 2, no. 5, pp. 211-263, 1973.

[15] I. L. Chenchev, V. V. Ivanova, D. Z. Neychev, and R. B. Cholakova, "Application of platelet-rich fibrin and injectable platelet-rich fibrin in combination of bone substitute material for alveolar ridge augmentatio-a case report," Folia Medica, vol. 59, no. 3, pp. 362-366, 2017.

[16] S. Serratì, F. Margheri, S. Bruschi et al., "Plasminogen activators and inhibitor type-1 in alveolar osteitis," European Journal of Oral Sciences, vol. 114, no. 6, pp. 500-503, 2006.

[17] P. A. Lone, S. W. Ahmed, V. Prasad, and B. Ahmed, "Role of turmeric in management of alveolar osteitis (dry socket): a randomised clinical study," Journal of Oral Biology and Craniofacial Research, vol. 8, no. 1, pp. 44-47, 2018.

[18] M. Taberner-Vallverdu, M. Nazir, M. Sanchez-Garces, and C. Gay-Escoda, "Efficacy of different methods used for dry socket management: a systematic review," Medicina Oral 
Patología Oral Y Cirugia Bucal, vol. 20, no. 5, pp. e633-e639, 2015.

[19] M. A. Rauf, A. Kamal, and S. Farooq, "Management of dry socket: hydrogen peroxide as an irrigant," Pakistan Journal of Medical and Health Sciences, vol. 8, pp. 772-773, 2014

[20] E. Tanaka, S. Kuroda, S. Horiuchi, A. Tabata, and T. El-Bialy, "Low-intensity pulsed ultrasound in dentofacial tissue engineering," Annals of Biomedical Engineering, vol. 43, no. 4, pp. 871-886, 2015.

[21] G. Jovanovic, N. Buric, N. Krunic, M. Tijanic, and S. Stojanovic, "Assessment of the effectiveness of low level laser in the treatment of alveolar osteitis," Vojnosanitetski Pregled, vol. 68, no. 6, pp. 506-510, 2011.

[22] M. Eshghpour, F. Ahrari, N. Najjarkar, and M. Khajavi, "Comparison of the effect of low level laser therapy with alvogyl on the management of alveolar osteitis," Medicina Oral Patología Oral Y Cirugia Bucal, vol. 20, no. 3, pp. e386-e392, 2015.

[23] J. Ahmedi, E. Ahmedi, O. Sejfija, Z. Agani, and V. Hamiti, "Efficiency of gaseous ozone in reducing the development of dry socket following surgical third molar extraction," European Journal of Dentistry, vol. 10, no. 3, pp. 381-385, 2016.

[24] A. R. Khan and J. Abid, "Management of dry socket using ozone gel vs. alvogyl-prospective clinical trial," International Dental Journal of Students Research, vol. 3, pp. 29-33, 2015.

[25] P. A. Efron and L. L. Moldawer, "Cytokines and wound healing: the role of cytokine and anticytokine therapy in the repair response," Journal of Burn Care \& Rehabilitation, vol. 25, no. 2, pp. 149-160, 2004.

[26] M. O. Schär, J. Diaz-Romero, S. Kohl, M. A. Zumstein, and D. Nesic, "Platelet-rich concentrates differentially release growth factors and induce cell migration in vitro," Clinical Orthopaedics and Related Research, vol. 473, no. 5, pp. 1635-1643, 2015.

[27] R. Jin, G. Song, J. Chai, X. Gou, G. Yuan, and Z. Chen, "Effects of concentrated growth factor on proliferation, migration, and differentiation of human dental pulp stem cells in vitro," Journal of Tissue Engineering, vol. 9, 2018.

[28] A. S. Plachokova, D. Nikolidakis, J. Mulder, J. A. Jansen, and N. H. J. Creugers, "Effect of platelet-rich plasma on bone regeneration in dentistry: a systematic review," Clinical Oral Implants Research, vol. 19, no. 6, pp. 539-545, 2008.

[29] Y. Xu, J. Qiu, Q. Sun et al., "One-year results evaluating the effects of concentrated growth factors on the healing of intrabony defects treated with or without bone substitute in chronic periodontitis," Medical Science Monitor, vol. 25, pp. 4384-4389, 2019.

[30] H. Mohan, J. Ryan, B. Whelan, and A. Wakai, "The end of the line? the visual analogue scale and verbal numerical rating scale as pain assessment tools in the emergency department," Emergency Medicine Journal, vol. 27, no. 5, pp. 372-375, 2010.

[31] F. Ghaderi, S. Banakar, and S. Rostami, "Effect of pre-cooling injection site on pain perception in pediatric dentistry: "a randomized clinical trial"” Dental Research Journal, vol. 10, no. 6, p. 790, 2013.

[32] A. Haraji, E Lassemi, M. H. Motamedi, M. Alavi, and S. Adibnejad, "Effect of plasma rich in growth factors on alveolar osteitis," National Journal of Maxillofacial Surgery, vol. 3, no. 1, pp. 38-41, 2012.

[33] J. L. Rutkowski, D. A. Johnson, N. M. Radio, and J. W. Fennell, "Platelet rich plasma to facilitate wound healing following tooth extraction," Journal of Oral Implantology, vol. 36, no. 1, pp. 11-23, 2010.
[34] A. Lubkowska, B. Dolegowska, and G. Banfi, "Growth factor content in PRP and their applicability in medicine," Journal of Biological Regulators and Homeostatic Agents, vol. 26, no. 2, pp. 3-22, 2012.

[35] B. T. Khan, M. N. Kiani, M. H. B. Saeed, and A. Z. Khan, "Risk factors assessment for dry sockets: a logistic regression analysis study," Journal of Oral and Maxillofacial Surgery, Medicine, and Pathology, vol. 27, no. 6, pp. 753-756, 2015.

[36] N. Kanwal, J. Muttappally Myalil, S. Al-Sharbatti, and I. Ismail, "Contraceptive utilisation among mothers of reproductive age in ajman, United Arab Emirates," Sultan Qaboos University Medical Journal, vol. 17, no. 1, pp. e50-58, 2017.

[37] J. E. Catellani, S. Harvey, S. H. Erickson, and D. Cherkin, "Effect of oral contraceptive cycle on dry socket (localized alveolar osteitis)," Journal of the American Dental Association, vol. 101, no. 5, pp. 777-780, 1980.

[38] C. Upadhyaya and M. Humagain, "Prevalence of dry socket following extraction of permanent teeth at Kathmandu university teaching hospital (KUTH), dhulikhel, kavre, Nepal: a study," Kathmandu University Medical Journal, vol. 8, no. 1, pp. 18-24, 2010.

[39] D. Nitzan, J. F. Sperry, and T. D. Wilkins, "Fibrinolytic activity of oral anaerobic bacteria," Archives of Oral Biology, vol. 23, no. 6, pp. 465-470, 1978.

[40] M. H. A. Karbassi, R. Salehi, K. Kheirollahi, M. G. Targhi, M. J. Sadrabad, and B. Yousefipour, "The relationship between socket blood sugar and post-extraction complications in type II diabetic and non-diabetic patients," Iranian Journal of Diabetes and Obesity, vol. 7, no. 1, p. 13, 2015.

[41] M. H. Abu Younis and R. O. Abu Hantash, "Dry socket: frequency, clinical picture, and risk factors in a palestinian dental teaching center," The Open Dentistry Journal, vol. 5, no. 1, pp. 7-12, 2011.

[42] M. Al-Houqani, A. L. Jabari, A. A. Naeemi et al., "Patterns of tobacco use in the United Arab Emirates healthy future (UAEHFS) pilot study," PloS One, vol. 13, no. 5, Article ID e0198119, 2018.

[43] B. Tarakji, L. A. Saleh, A. Umair, S. N. Azzeghaiby, and S. Hanouneh, "Systemic review of dry socket: aetiology, treatment, and prevention," Journal of Clinical and Diagnostic Research, vol. 9, no. 4, p. ZE10, 2015.

[44] S. Chakravarthi, "Platelet rich fibrin in the management of established dry socket," Journal of the Korean Association of Oral and Maxillofacial Surgeons, vol. 43, no. 3, pp. 160-165, 2017.

[45] I. Chenchev, D. Dobreva, V. Ivanova et al., "Treatment of dry socket with platelet-rich fibrin," Journal of IMA-Annual Proceeding (Scientific Papers), vol. 23, no. 3, pp. 1702-1705, 2017.

[46] H. Masuki, T. Okudera, T. Watanebe et al., "Growth factor and pro-inflammatory cytokine contents in platelet-rich plasma (PRP), plasma rich in growth factors (PRGF), advanced platelet-rich fibrin (A-PRF), and concentrated growth factors (CGF)," International Journal of Implant Dentistry, vol. 2, no. 1, p. 19, 2016.

[47] E. M. King, T. L. Cerajewska, M. Locke, N. C. A. Claydon, M. Davies, and N. X. West, "The efficacy of plasma rich in growth factors for the treatment of alveolar osteitis: a randomized controlled trial," Journal of Oral and Maxillofacial Surgery, vol. 76, no. 6, pp. 1150-1159, 2018.

[48] L. Fortunato, F. Bennardo, C. Buffone, and A. Giudice, "Is the application of platelet concentrates effective in the prevention and treatment of medication-related osteonecrosis of the jaw? 
A systematic review," Journal of Cranio-Maxillofacial Surgery, vol. 48 , no. 3, 2020.

[49] A. Giudice, S. Barone, C. Giudice, F. Bennardo, and L. Fortunato, "Can platelet-rich fibrin improve healing after surgical treatment of medication-related osteonecrosis of the jaw? a pilot study," Oral Surgery, Oral Medicine, Oral Pathology and Oral Radiology, vol. 126, no. 5, pp. 390-403, 2018. 